\title{
Atuação do Fisioterapeuta em um programa de residência multiprofissional em saúde em Santarém, Oeste do Pará, Amazônia
}

\author{
Perspectives and Challenges of the Physiotherapy in a multiprofessional residency program in \\ health in Santarém, Western Pará, Amazonia
}

Desempeño del fisioterapeuta en un programa multidisciplinario de residencia en salud en Santarém, Extremo Oeste Pará, Amazonía

\author{
Bruna Luiza da Silva Barbosa \\ ORCID: https://orcid.org/0000-0003-3613-9488 \\ Universidade do Estado do Pará, Brasil \\ E-mail: brunalsb82@gmail.com \\ Livia de Aguiar Valentim \\ ORCID: https://orcid.org/0000-0003-4255-8988 \\ Universidade do Estado do Pará, Brasil \\ E-mail: livia.valentim@uepa.br \\ Daliane Ferreira Marinho \\ ORCID: https://orcid.org/0000-0003-3849-1215 \\ Universidade do Estado do Pará, Brasil \\ E-mail: daliane.marinho@uepa.br \\ Nádia Vicência do Nascimento Martins \\ ORCID: https://orcid.org/0000-0002-8166-644X \\ Universidade do Estado do Pará, Brasil \\ E-mail: nadia.martins@uepa.br \\ Sheyla Mara Silva de Oliveira \\ ORCID: https://orcid.org/0000-0001-6666-2363 \\ Universidade do Estado do Pará, Brasil \\ E-mail: sheylaoliveira@uepa.br \\ Marina Smidt Celere Meschede \\ ORCID: https://orcid.org/0000-0002-6519-9466 \\ Universidade Federal do Oeste do Pará, Brasil \\ E-mail: marcelere@yahoo.com.br \\ Teógenes Luiz Silva da Costa \\ ORCID: https://orcid.org/0000-0001-7040-7939 \\ Universidade Federal do Oeste do Pará, Brasil \\ E-mail: teogeneslsc@yahoo.com.br
}

\begin{abstract}
Resumo
A Residência Multiprofissional em Saúde (RMS) é uma modalidade de pós graduação que oferece uma formação teórico prática latu sensu, com o intuito de formar especialistas de diversas áreas profissionais da saúde com capacidade para promover ações integradas e visando a prevenção, promoção e reabilitação dos pacientes. $O$ presente artigo tem como objetivo relatar as perspectivas e os principais desafios de um profissional fisioterapeuta inserido no Programa de Residência Multiprofissional em Atenção Integral em Traumatologia e Ortopedia vinculado a Universidade do Estado do Pará (UEPA). Este estudo consiste em um relato descritivo de experiência de caráter retrospectivo, da inserção e atuação de uma fisioterapeuta no ambulatório de fisioterapia de um Hospital Regional, com atividades realizadas no período de março a maio de 2021. As perspectivas sobre o programa de RMS é que forme um profissional com formação humanista, generalista assim como estar responsável por atuar de forma crítica, reflexiva, com autonomia, responsabilidade, conduta ética, além de rigor científico e intelectual na área de traumatologia e ortopedia. Entretanto, os desafios enfrentados nesses primeiros meses vão desde a pouca atuação multiprofissional no ambulatório do HRBA até a extensa carga horária teórico- prática, perpassando pela falta de disciplina específica no primeiro ano de residência. Conclui-se que apesar da sobrecarga causada ao residente por conta da extensa carga horária do programa, a residência multiprofissional em ortopedia e traumatologia proporciona uma vivência teórico prática que enriquece o leque de experiência do profissional, contribuindo de forma efetiva na assistência ao paciente.
\end{abstract}

Palavras-chave: Fisioterapeutas; Ortopedia; Traumatologia; Assistência integral à saúde. 


\begin{abstract}
The Health Multiprofessional Residency Program (HMRP) is a postgraduate modality (Lato Sensu), whose offers a theoretical and practical training, in order to train specialists from different professional areas of health with the capacity to promote integrated actions and aiming at the prevention, promotion and rehabilitation of patients. This article seeks to report the perspectives and main challenges of a professional physiotherapist inserted in the Multiprofessional Residency Program in Comprehensive Care in Traumatology and Orthopedics linked to the University of the State of Pará (UEPA). This is a descriptive report of a retrospective experience, of the insertion and performance of a physiotherapist in the physiotherapy clinic of a Regional Hospital, with activities carried out from March to May 2021. The perspectives about the HMRP are that it trains a professional with an humanista and generalist training, as well as makes him capable to being responsible for acting in a critical and reflective way, with autonomy, responsibility, ethical conduct, as well as scientific and intellectual rigor in the area of traumatology and orthopedics. However, the challenges faced in these first months range from the lack of multidisciplinary work at the HRBA outpatient clinic to the extensive theoretical and practical workload, including the lack of specific discipline in the first year of residency. It is concluded that despite the burden caused to the resident due to the extensive workload of the program, the multidisciplinary residency in orthopedics and traumatology provides a practical theoretical experience that enriches the professional's range of experience, effectively contributing to patient care.
\end{abstract}

Keywords: Physiotherapists; Orthopedics; Traumatology; Comprehensive health care.

\title{
Resumen
}

La Residencia Multiprofesional en Salud (RMS) es una modalidad de postgrado que ofrece una formación teóricopráctica latu sensu, con la intención de formar especialistas en diferentes áreas profesionales de la salud con capacidad de promover acciones integradas y encaminadas a la prevención, promoción y rehabilitación de los pacientes. El presente artículo tiene como objetivo relatar las perspectivas y los principales desafíos de un profesional fisioterapeuta insertado en el Programa de Residencia Multiprofesional en Atención Integral en Traumatología y Ortopedia vinculado a la Universidade do Estado do Pará (UEPA). Este estudio consiste en un informe descriptivo de experiencia retrospectiva de la inserción y actuación de un fisioterapeuta en la consulta externa de fisioterapia de un Hospital Regional, con actividades realizadas de marzo a mayo de 2021. Las perspectivas del programa IMR son que forme un profesional con educación humanista y generalista, además de ser responsable de actuar de manera crítica y reflexiva, con autonomía, responsabilidad, conducta ética, además de rigor científico e intelectual en el área de traumatología y ortopedia. Sin embargo, los retos a los que se enfrentan en estos primeros meses van desde el escaso trabajo multidisciplinar en la consulta externa del HRBA hasta la extensa carga de trabajo teórico y práctico, pasando por la falta de disciplina específica en el primer año de residencia. Se concluye que a pesar de la sobrecarga provocada al residente por la extensa carga de trabajo del programa, la residencia multiprofesional en ortopedia y traumatología proporciona una experiencia teórico-práctica que enriquece el abanico de experiencias profesionales, contribuyendo eficazmente a la atención del paciente.

Palabras clave: Fisioterapeutas; Ortopedia; Traumatología; Atención integral de salud.

\section{Introdução}

Os programas de residência multiprofissional em saúde foram instituídos pelo Ministério da Saúde a partir da Lei $\mathrm{n}^{\circ}$ 11.129, de 30 de junho de 2005 e são considerados uma formação na modalidade de pós-graduação lato sensu, voltados para profissionais da saúde, não médicos, tendo como principal característica o ensino em serviço de acordo com os princípios e diretrizes do cuidado no âmbito do SUS, tendo supervisão e orientação de profissionais éticos e qualificados, com carga horária semanal 60 horas (Brasil, 2006). Dessa maneira o ensino em serviço busca a produção do conhecimento através do dia a dia dos profissionais, e assim melhorar a qualidade da assistência e do atendimento ao usuário do Sistema Único de Saúde (SUS) (Arnemann et al, 2021).

Segundo o Ministério da Saúde (2006), as residências multiprofissionais em saúde proporcionam aos residentes habilidades para atuarem na assistência durante os seus dois anos de formação, voltada para uma atenção integral à saúde e na gestão do cuidado. Além disso, os programas de residência devem destinar $80 \%$ da sua carga horária total para as atividades práticas, ou seja, nos equipamentos públicos de saúde o que possibilitam o contato rotineiro com os usuários e outros profissionais que integram a equipe multiprofissional, proporcionando uma vivência extremamente relevante nos dias atuais.

$\mathrm{Na}$ cidade de Santarém, atualmente existem três Programas de Residência Multiprofissional vinculados às duas maiores universidades da região Oeste do Pará: a Universidade Estadual do Pará (UEPA) e a Universidade Federal do Oeste do Pará (UFOPA). Dentre os Programas, aponta-se para o de Traumatologia e Ortopedia vinculado a UEPA e tem como objetivo 
geral formar especialistas em ortopedia e traumatologia, com visão holística do paciente e capaz de promover ações integradas no atendimento ambulatorial, domiciliar, hospitalar, como também no âmbito da saúde do paciente politraumatizado em potencial (UEPA e HRBA, 2014).

A partir de 2014, a UEPA e o Hospital Regional do Baixo Amazonas (HRBA) ofertaram a sua primeira turma e o programa de residência em Traumatologia e Ortopedia que conta hoje com diferentes categorias profissionais como enfermeiros, terapeutas ocupacionais, psicólogos, assistentes sociais, farmacêuticos e fisioterapeutas (UEPA e HRBA, 2014).

De acordo com Maia et al (2015) e Oliveira e Medeiros (2018), a inserção do fisioterapeuta no programa se faz relevante visto que as afecções traumato-ortopédicas são um importante problema de saúde pública, ocasionando sérias alterações funcionais e devido a isso a reabilitação fisioterapêutica vem ganhando cada vez mais espaço nos últimos tempos, pois esta é fundamental para o restabelecimento da capacidade funcional dos indivíduos acometidos. Mas para além de ações curativas e de reabilitação, a fisioterapia atua na prevenção de agravos e doenças e na promoção de saúde, portanto se faz necessária a presença do Fisioterapeuta também na atenção primária e não apenas na média e na alta complexidade.

Berlato, Tokumoto e Oliveira (2009), Nogueira, Costa, Arruda, Leite e Sousa (2016) e Conselho Federal de Fisioterapia e Terapia Ocupacional (COFFITO, 2020) afirmam que a fisioterapia traumato ortopédica é a área que atua na prevenção, promoção e tratamento dos diversos acometimentos que abrangem o sistema osteomioarticular, objetivando, principalmente, o reestabelecimento da funcionalidade do paciente, se utilizando de diversos recursos da fisioterapia, que vão desde exercícios terapêuticos até Práticas Integrativas complementares, e assim buscar que este tenha um retorno breve e independente a suas atividades de vida diária.

O fisioterapeuta destina uma abordagem personalizada ao paciente, respeitando as limitações e as particularidades específicas de cada indivíduo. Consequentemente, com um tratamento de qualidade, o indivíduo começa a apresentar mais independência para realizar suas atividades da vida diária tais como tarefas domésticas, atividades laborais e praticar esportes. Portanto, atualmente se torna indispensável o acompanhamento fisioterapêutico especializado (Maia et al, 2015; Nogueira, Costa, Arruda, Leite e Sousa, 2016; Oliveira e Medeiros, 2018; ).

Diante do contexto apresentado, este artigo tem como objetivo relatar as perspectivas e os principais desafios de um profissional fisioterapeuta no Programa de Residência Multiprofissional em atenção integral em ortopedia e traumatologia vinculado a UEPA/HRBA, na cidade de Santarém, Oeste do Pará, Amazônia.

\section{Metodologia}

O presente artigo é produto de um estudo do tipo qualitativo, baseado em uma pesquisa de campo (GIL, 1999) em ambiente de prestação de serviços em saúde, onde se desempenhou atividades referentes à residência aqui em pauta, organizado na forma de relato de experiência. Do ponto de vista metodológico, utilizamos a observação participante como principal ferramenta de coleta de dados, balizado, na dimensão teórica, pelo artifício conceitual denominado "descrição densa" (GEERTZ, 1989).

Para Geertz (1989), a pesquisa social tem como principal ferramenta descrever minuciosamente as redes de relações sociais que estruturam a existência do fenômeno em observação para em seguida realizar a interpretação dos fatos (dados) da realidade investigada. Assim, no presente texto, intencionou-se organizar o conjunto de informações observadas em campo e organizar em formato de relato de experiência, o mesmo é a nossa interpretação referente aos fatos observados.

\section{Resultados e Discussão}

\subsection{Atividades vinculadas à prática do residente em fisioterapeuta no HRBA}

Durante o primeiro ano de residência (R1), os residentes de todas as áreas realizaram o momento chamado integração 
no HRBA onde são apresentadas as normas e rotinas hospitalares juntamente com o regimento da residência multiprofissional, além de esclarecer possíveis dúvidas quanto aos serviços e demandas voltadas para a residência multiprofissional. Após essa atividade de adaptação inicial, os residentes iniciaram as atividades no ambulatório de fisioterapia do HRBA. O ambulatório de fisioterapia do HRBA recebe pacientes de outros setores do hospital como o de oncologia e, principalmente, pós- cirúrgicos que realizaram cirurgias ortopédicas e traumatológicas. Ele é composto de duas salas para a reabilitação em eletrotermofototerapia e cinesioterapia (8 macas de capacidade), uma sala de mecanoterapia, uma sala de diatermia e um consultório (UEPA e HRBA, 2014). Nesse primeiro momento, o residente é inserido no setor de destino de acordo com o programa, mas de antemão é apresentado a política do hospital, o perfil e a rotina do setor e posteriormente integrado à equipe, e em busca de desenvolver habilidades especificas, terá sempre o suporte de um profissional especialista na área ( Paiva et al, 2017).

Para melhor atender o público, contam com uma equipe multiprofissional composta por fisioterapeutas, fonoaudiólogos, assistente social, psicólogo, nutricionista e enfermeiros, tendo uma média de 1000 atendimentos/mês. Os pacientes cirúrgicos por motivos ortopédicos e traumatológicos ficam sob responsabilidade dos residentes no hospital escola, com a supervisão de um preceptor qualificado e específico da área (UEPA e HRBA 2014).

Apesar de o ambulatório de fisioterapia receber um grande fluxo de pacientes todo mês, no último ano em virtude da pandemia de covid-19, observou-se que a demanda tem diminuído, em razão da diminuição do número de cirurgias eletivas por conta do momento pandêmico pelo qual estamos passando. Além disso, houve restrição no atendimento a pacientes de 60 anos ou mais, com a condição de que não tenham qualquer tipo de comorbidades, para preservar os pacientes de infecções por agentes biológicos, especialmente pelo vírus da COVID-19.

Quanto ao perfil dos pacientes que realizaram atendimento por fisioterapeutas e residentes verificou-se a grande ocorrência durante os estágios de desordens de membro superior (fratura distal de rádio, fratura de cotovelo, fratura de carpos e metacarpos, síndrome do túnel do carpo) e de membro inferior (fratura de platô tibial, fratura de pilão tibial, fratura de fêmur e fratura de maléolos). No ambulatório de fisioterapia, existem atualmente dois preceptores que atuam em turnos diferentes, esclarecendo dúvidas específicas dos casos que estão sendo atendidos no ambulatório, realizando encontros da equipe dentro do horário do serviço para a discussão de casos clínicos, sempre em conjunto com os residentes da atenção em oncologia.

Por ser um ambulatório específico da Fisioterapia, é incomum a atuação em conjunto com profissionais de outras áreas. Porém, trabalhar ao lado de outro profissional favorece a qualidade do atendimento e uma melhor evolução do paciente, um exemplo disso é o acompanhamento juntamente a um profissional da psicologia, visto que, a saúde mental do paciente está diretamente ligada à saúde física. O terapeuta ocupacional é outro profissional de suma importância para a atuação em conjunto com o fisioterapeuta, seja em pacientes ortopédicos e traumatológicos, seja em pacientes oncológicos, pois, há uma importante melhora da funcionalidade das atividades de vida diária do paciente. Segundo Nascimento e Bulhões (2021), a falta de estrutura física, de interação entre os profissionais e a prática assistencial realizada de forma fragmentada por parte dos profissionais podem estar contribuindo para que essa prática integrativa tenha uma implementação lenta. Porém, o atendimento realizado de forma individualizada está dentro de um modelo antiquado e, portanto, faz-se necessário que a assistência seja prestada com mais qualidade e de maneira holística (Nascimento e Omena, 2021).

\subsection{Atividades vinculadas à teoria do residente em fisioterapeuta}

Quanto à formação teórica da residência, as disciplinas do eixo comum apresentam assuntos de grande valia à área da saúde, como "Gestão nos serviços de saúde" e outras, as quais nem sempre são abordadas de forma completa na graduação, no entanto, por vezes as disciplinas acabam ocorrendo ao mesmo tempo, e os residentes acabam por ficarem sobrecarregados com as várias aulas, atividades avaliativas, e ir para o serviço, o que muitas vezes ultrapassa às 60 horas semanais. Além disso, 
percebe-se uma carência de articulação entre a teoria e a prática, especialmente no primeiro ano de residência, pois as disciplinas são mais voltadas para a multiprofissionalidade. Gelbcke, Matos e Sallun (2012) e Pereira, Junior e Galvão (2018) asseguram que as disciplinas realizadas em conjunto com outros profissionais fortalecem a multiprofissionalidade e a interdisciplinaridade, fomentando o diálogo entre as categorias, seguindo os princípios e diretrizes do SUS e fortalecendo a assistência de acordo com a realidade social da região abrangida. Porém, nota-se a ausência de disciplinas voltadas para a especificidade de cada profissão, levando em conta que os residentes já se encontram na prática, o que corrobora com estudos recentes que identificaram as principais insatisfações de residentes, que são a extensa carga horária, a sobrecarga de trabalho, além, da falta de articulação entre a teoria e a prática, pois apresenta uma disparidade entre a formação teórica e a realidade da atuação nos serviços de saúde (Fernandes e colaboradores, 2017; Silva e Moreira, 2019). A sobrecarga aliada ao desgaste causa sofrimento ao residente durante o processo de formação uma vez que nem sempre estes conseguem atender todas as demandas que lhes são conferidas. Este desgaste emocional acarreta a perda do sono que ocasiona uma gama de consequências influenciando diretamente na qualidade de vida e na diminuição do rendimento no trabalho e na aprendizagem (Fernandes et al, 2017; Silva e Moreira, 2019; Silva e Dallbelo, 2019; Artico et al, 2020; Moura, Santos e Barroso, 2021).

\section{Considerações Finais}

Dado o exposto, percebe-se que a residência multiprofissional causa sobrecarga nos residentes devido à extensa carga horária teórica e prática, o que acaba por ocasionar angústia no processo de formação profissional, uma vez que o profissional/aluno não consegue realizar todas as atribuições que lhe são repassadas. No entanto a residência proporciona a atualização e o aperfeiçoamento das competências e habilidades técnicas do profissional através da integração e da vivência de saberes que se é experimentado no dia a dia do serviço aliado à formação teórica da área de Traumatologia e Ortopedia, enriquecendo e contribuindo de forma efetiva na assistência ao paciente. Dessa forma, se faz necessário que novos estudos relacionados ao tema sejam realizados para que se os programas de residência sejam aprimorados.

\section{Referências}

Arnemann, C. T., Kruse, M. H. L., Silva, M. E. K., Terra, M. G., Mello, A. de L., Silva, D. T. da, \& Souto, V. T. (2021). Sonhos de preceptores para um programa de residência multiprofissional: Utopias a serem investidas? Research, Society and Development, $10(7)$, e27010716079. https://doi.org/10.33448/rsd-v10i7.16079

Artico, G. A., Soares Rezende Lopes, M. T., Santos Ferreira de Omena, M. B., Fernandes Cardoso da Silva Rodrigues, T., Bellini Cardoso, L. C., Trindade Radovanovic, C. A., \& Lopes de Moraes Gil, N. (2020). <b\&gt, Percepções de residentes em urgência e emergência sobre sua formação: desafios para a aprendizagem / Perceptions of residents in urgency and emergency about their training: challenges for learning. Ciência, Cuidado E Saúde, 19. https://doi.org/10.4025/cienccuidsaude.v19i0.50149

Berlato, E., Tokumoto, L. F., \& Oliveira, M. V. Papel da fisioterapia em lesões traumato ortopédicas de quadril em idosos. 2009. 49F. Monografia (Especialização em Fisioterapia Traumato Ortopedica Funcional) - UNISALESIANO- Centro

Brasil. Ministério da Saúde. Secretaria de Gestão do Trabalho e da Educação na Saúde. Departamento de Gestão da Educação na Saúde. Residência multiprofissional em saúde: experiências, avanços e desafios / Ministério da Saúde, Secretaria de Gestão do Trabalho e da Educação na Saúde, Departamento de Gestão da Educação em Saúde. Ministério da Saúde, 2006.

Casanova, I. A., Batista, N. A., \& Ruiz-Moreno, L. (2018). A Educação Interprofissional e a prática compartilhada em programas de residência multiprofissional em Saúde. Interface, 22, 1325-1337. doi.org/10.1590/1807-57622017.0186.

Conselho Federal de Fisioterapia e Terapia Ocupacional (2020). Traumato-Ortopedia na Atenção Primária. https://www.coffito.gov.br/nsite/?p=15716

Fernandes, M., Beck, C., Weiller, T., Coelho, A., Prestes, F., \& Donaduzzi, D. Satisfação E Insatisfação De Residentes Multiprofissionais Em Saúde Na Perspectiva Da Formação. Revista Baiana de Enfermagem. 31. 10.18471/rbe.v31i3.18344.

Gelbcke, L. F., Matos, E., \& Sallun, C. N. (2012). Desafios para a integração multiprofissional e interdisciplinar. Revista Eletrônica Tempus - Acta de Saúde Coletiva, 6, 31-39.

Geertz, C. A interpretação das culturas. LTC, 1989.

Gil, A. C. Métodos e técnicas de pesquisa social. Atlas, 1999. 
Research, Society and Development, v. 10, n. 12, e433101220709, 2021

(CC BY 4.0) | ISSN 2525-3409 | DOI: http://dx.doi.org/10.33448/rsd-v10i12.20709

Maia F., Moura, E., Madeiros, E., Carvalho, R., Silva, S., \& Santos, G. (2015). A importância da inclusão do profissional fisioterapeuta na atenção básica de Saúde. Revista da Faculdadede Ciências Médicas de Sorocaba, 17(3), 110-115.

Moura, F. J. L. de, Santos, D. N. R. dos, \& Barroso, B. I. de L. (2021). Trabalhe enquanto eles dormem? A qualidade do sono dos residentes de Programas de Residência Multiprofissional em Saúde. Research, Society and Development, 10(9), e44910910976. https://doi.org/10.33448/rsd-v10i9.10976

Nascimento, A. C. B., do, \& Omena, K. V. M. (2021). A Educação Interprofissional em Programas de Residência Multiprofissional em Saúde no Brasil: Uma revisão integrativa. Research, Society and Development, 10, e8010413655, 10.33448/rsd-v10i4.13655. https://rsdjournal.org/index.php/rsd/article/view/13655.

Nascimento, A. C. B., \& Bulhões, K. V. M. (2021). Educação Interprofissional em Programas de Residência Multiprofissional em Saúde no Brasil: Uma revisão integrativa. Research, Society and Development, 10 http://dx.doi.org/10.33448/rsd-v10i4.13655

Nogueira, A. F., da Costa B. H., de Arruda, E. F., Leite, M. B., \& de Sousa, C. S. (2016). Principais distúrbios traumato-ortopédicos atendidos em clínicasescola de fisioterapia. Rev Cient Fac Educ e Meio Ambient [Internet],7(2):33-44.

Oliveira, A. M. B., \& Medeiros, N. T. Fisioterapia na residência multiprofissional em Saúde da Família: relato de Experiência. Sanare, Sobral, $17,91-99$.

de Paiva, D. R., Guimarães, V. S., Rôla, Q. C. S., de Castro, I. P. R., Gomes, K. S., \& dos Anjos, J. L. M. (2017). Inserção e atuação de fisioterapeutas residentes em um serviço de emergência hospitalar: relato de experiência. Revista Pesquisa Em Fisioterapia, 7(2), 255-260. https://doi.org/10.17267/22382704rpf.v7i2.1351

Pereira, M. N. S., Junior, J. C. M. N., \& Galvão, E. F. C. (2018). A visão dos preceptores a respeito da residência multiprofissional com foco na ortopedia e traumatologia no interior da amazônia. Revista EXITUS, 8(1),332-360. https://www.redalyc.org/articulo.oa?id=553159820014

Projeto da Residência Multiprofissional na Atenção Integral em Ortopedia e Traumatologia. 2014.

Silva, C. A., \& Dalbello-Araujo, M. (2019). Programa de Residência Multiprofissional em Saúde: o que mostram as publicações. Saúde em Debate [online]. 43, 1240-1258. https://doi.org/10.1590/0103-1104201912320.

Silva, R. M. B., \& Moreira, S. N. T. (2021). Estresse e Residência Multiprofissional em Saúde: Compreendendo Significados no Processo de Formação. Revista Brasileira de Educação Médica. 43, 157-166. https://doi.org/10.1590/1981-52712015v43n4RB20190031. 